\title{
A GEOMETRICAL APPROACH TO THE JACOBIAN CONJECTURE FOR $n=2$
}

\author{
By Lê Dũng Tráng and Claude Weber
}

Let us recall the Jacobian conjecture (see [B-C-W] §I p. 288):

Jacobian Conjecture. Let $F: \mathbf{C}^{n} \rightarrow \mathbf{C}^{n}$ be a polynomial map. Suppose that, for every point $x \in \mathbf{C}^{n}$, the derivative $F^{\prime}(x)$ is invertible. Then the map $F$ is invertible.

In this lecture we describe a geometrical approach to this conjecture when $n=2$.

First we shall need some results on the geometry of complex polynomial functions.

\section{Topology of polynomial functions.}

It is known that a complex polynomial function might not be a locally trivial topological fibration over the complement in $\mathbf{C}$ of its critical values. As an example, consider the polynomial $f(X, Y)=X-X^{2} Y$ (see [B]): this complex polynomial function has no critical point, but it is not a locally trivial topological fibration on C. In fact the fiber $f=0$ has two connected components, but, for any $\lambda \neq 0, f=\lambda$ has only one component. However there is a general theorem of $\mathrm{R}$. Thom:

Theorem (Тном). Let $f: \mathbf{C}^{n} \rightarrow \mathbf{C}$ be a complex polynomial function. There is a minımal finıte set $A(f)$, such that $f$ induces a locally trivial topological fibration over the complement of $A(f)$ in $\mathbf{C}$.

One can check that the finite set $A(f)$ always contains the set of critical values $D(f)$. Let us write:

$$
A(f)=D(f) \cup I(f)
$$

where $D(f)$ and $I(f)$ might not be disjoint. By definition $I(f) \neq \emptyset$ depends on "accidents" at $\infty$. We shall call $A(f)$ the set of atypical values of $f$.

Polynomals which are locally trivial topological fibrations are well understood because of the following observation:

Proposition. If the complex polynomial function

$$
f: \mathbf{C}^{2} \rightarrow \mathbf{C}
$$

is a locally trivial fibration on $\mathbf{C}$, there is an algebraic automorphism $\sigma$ of the complex plane, such that $f \circ \sigma=X$.

Received April 26, 1994. 
This proposition is consequence of the Embedding Theorem of S. Abhyankar and T.T. Moh ([A-M]):

Theorem (S. AbhyANKAR - T.T. MoH). Let $C$ be a complex algebraic affine curve embedded in the complex plane $\mathrm{C}^{2}$ and isomorphic to the complex line $\mathbf{C}$. Let $f=0$ be its (reduced) equation. Then, there is an algebraic automorphism $\sigma$ of the complex plane, such that $f \circ \sigma=X$, where $X$ is one of the coordinates of $\mathbf{C}^{2}$.

The methods developed in this note are the basic tool for a geometrical proof of the Abhyankar-Moh Theorem (see [A]).

The proposition above has the following consequence:

Corollary. Let $F=(f, g): \mathbf{C}^{2} \rightarrow \mathbf{C}^{2}$ be a polynomial map. Suppose that, for every point $x \in \mathbf{C}^{2}$, the derivative $F^{\prime}(x)$ is invertible and furthermore, suppose that $f$ is a locally trivial topological fibration on $\mathrm{C}$. Then $\mathrm{F}$ is invertible.

Proof. If we assume that $f$ is a locally trivial topological fibration on $\mathbf{C}$, the general fibers of $f$ are isomorphic to the complex line. Therefore the Embedding Theorem of S. Abhyankar and T.T. Moh shows that there is an algebraic automorphism $\sigma$ of the complex plane such that $f \circ \sigma=X$. This proves the proposition above. Now the Jacobian $J(F \circ \sigma)$ of $F \circ \sigma$ is the product of the Jacobians $J(F)$ and $J(\sigma)$ and therefore is equal to a non-zero constant $k$. But $F \circ \sigma=(X, g \circ \sigma)$, so that, by integration

$$
g \circ \sigma=k Y+h(X)
$$

where $h$ is a complex polynomial function. Hence $F \circ \sigma$ is an automorphism of the complex plane, which implies that $F$ is also an automorphism of the complex plane, as announced.

As a consequence, the Jacobian conjecture will be proved, if one can show that:

Conjecture. Suppose that $f: \mathbf{C}^{2} \rightarrow \mathbf{C}$ is not a locally trivial topological fibration on C. Then, for any complex polynomial function $g$, the Jacobian $J(f, g)$ cannot be a non-zero constant.

We have seen that $f: \mathbf{C}^{2} \rightarrow \mathbf{C}$ is not locally trivial topological fibration on $\mathbf{C}$, if and only if $A(f)$ is non-empty. If the set of critical values is non-empty, our conjecture is obviously true. Therefore we shall assume that the polynomial $f$ has no critical point. We are led to understand the meaning of $I(f) \neq \emptyset$. In $[\mathrm{H}-\mathrm{L}]$, we give a way to calculate $I(f)$. Now we describe how to do it.

Let $f(x, y)$ be the complex polynomial of degree $d$ which defines $f$ :

$$
f(x, y)=\sum_{\alpha+\beta \leqq d} c_{\alpha, \beta} x^{\alpha} y^{\beta} .
$$

The homogeneization of $f(X, Y)$ is the homogeneous complex polynomial $F(X, Y, T)$ of degree $d$ :

$$
F(X, Y, T)=\sum_{\alpha+\beta \leqq d} c_{\alpha, \beta} X^{\alpha} Y^{\beta} T^{d-\alpha-\beta}
$$


The compactification of the fibers $f=\lambda$ in the complex projective plane $\mathbf{P}^{2}$ are the projective curves $C_{\lambda}$ with projective equations $F-\lambda T^{d}=0$. These curves pass through the same points on the line at infinity $T=0$, namely through:

$$
\left\{x_{1}, \ldots, x_{r}\right\}=\left\{F=T^{d}=0\right\}
$$

which corresponds to the asymptotic directions of the level curves $f=\lambda$.

For each $x_{i}, 1 \leqq i \leqq r$, there is an integer

$$
\mu_{\imath}:=\inf _{\{\lambda \in \mathbf{C}\}} \mu\left(C_{\lambda}, x_{i}\right)
$$

where $\mu\left(C_{\lambda}, x_{i}\right)$ is the Milnor number of the curve $C_{\lambda}$ at the point $x_{i}$. Now we can state:

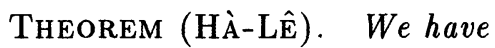

$$
I(f)=\left\{\lambda \in \mathbf{C} \mid \text { there } \text { ss } 1 \leqq i \leqq r \text { such that, } \mu\left(C_{\lambda}, x_{i}\right) \neq \mu_{i}\right\} .
$$

For example, consider $f(X, Y)=X-X^{2} Y$. Then

$$
F(X, Y, T)=X T^{2}-X^{2} Y
$$

and the asymptotic directions of the level curves $f=\lambda$ are the points $x_{1}:=(1: 0: 0)$ and $x_{2}:=(0: 1: 0)$. At $x_{1}$, we have $\mu_{1}=0$ and the Milnor number of all the curves $C_{\lambda}$ is 0 , for all $\lambda \in \mathbf{C}$. At $x_{2}$, we have $\mu_{2}=2$ and the Milnor number of $C_{0}$ at $x_{2}$ is 3 . In this case $A(f)=I(f)=\{0\}$.

\section{Compactification of polynomial functions.}

In general it is easier to deal with proper maps. In the case of complex polynomial functions in two variables, there is a natural way to compactify the function. We consider the rational function $F / T^{d}$ on the complex projective plane $\mathbf{P}^{2}$. The set where $F=T^{d}=$ 0 is the set of indeterminacy of the rational function $F / T^{d}$. By blowing-up points, one can remove the indeterminacy of the rational function $F / T^{d}$. We shall describe a minimal way to do it.

- First fix $\lambda_{0} \notin A(f)$. Let $q: \mathcal{Y} \rightarrow \mathbf{P}^{2}$ be the embedded resolution of the projective curve $C_{\lambda_{0}}$. Let $L$ be a linear form which does not vanish at the points $x_{1}, \ldots, x_{r}$. For each component $D$ of the divisor

$$
D_{\infty}(\mathcal{Y}):=q^{-1}(T=0)
$$

we denote by $\nu_{D}\left(F / L^{d}\right)$ the multiplicity of $F / L^{d} \circ q$ along $D$. Now consider the points of indeterminacy of $F / T^{d} \circ q$ which might remain on $\mathcal{Y}$. Each of these points $\xi$ belongs to a component $D_{\xi}$ of the divisor $D_{\infty}(\mathcal{Y})$. Now at every point $\xi$ of indeterminacy of $F / T^{d} \circ q$ on $\mathcal{Y}$, perform point blowing-ups so that to separate non-singular branches having an intersection number equal to $\nu_{D_{\xi}}\left(T^{d} / L^{d}\right)-\nu_{D_{\xi}}\left(F / L^{d}\right)$ at $\xi$. In this way we obtain a map $p: \mathcal{X} \rightarrow \mathcal{Y}$. Denote $D_{\infty}(\mathcal{X}):=(q \circ p)^{-1}(T=0)$. We have:

TheOREM. On $\mathcal{X}, F / T^{d} \circ q \circ p$ defines a rational map onto $\mathbf{P}^{1}$. The restriction of this map to $\mathcal{X}-D_{\infty}(\mathcal{X})$ induces a map into $\mathbf{C}$ ısomorphic to $f$ by $\pi:=q \circ p$.

The map $\pi$ is minimal in the following sense: 
OBservation. The only components of the divisor $D_{\infty}(\mathcal{X}):=\pi^{-1}(T=0)$ which may have self-intersection -1 are some of the components $D$ such that the restriction of $F / T^{d} \circ \pi$ to $D$ is not constant and, possibly, the strict transform of $T=0$ by $\pi$ in $\mathcal{X}$.

Note that Vitushkin (see [V] Introduction) has used the existence of a non-singular compactification of $f$ in relation with the Jacobian conjecture when $n=2$.

For convenience, we shall call $\pi$ the minimal compactification of $f$. In fact the modification $\pi$ has interesting properties. First the intersection graph of the divisor $D_{\infty}(\mathcal{X})$ is a tree $\mathcal{A}$. Furthermore a careful study (using results of [L-M-W]) leads to the following key result:

Connectedness Theorem. The space $\left(F / T^{d} \circ \pi\right)^{-1}(\infty)$ is connected.

In other words the divisor $\left(F / T^{d} \circ \pi\right)^{-1}(\infty)$ defines a strict connected subtree $\mathcal{A}_{\infty}$ of $\mathcal{A}$. If the general fiber of $F / T^{d}$ o $\pi$ is connected, this theorem is actually an immediate consequence of Zariski Main Theorem (cf [Mu Theorem 3.24 and the footnote p. 52).

Let $\varphi:=F / T^{d} \circ \pi$. We shall call the divisor $D_{\infty}(\mathcal{X})$, the divisor at infinity of $\mathcal{X}$, and a component of $D_{\infty}(\mathcal{X})$ on which $\varphi$ is not constant is called dicritical.

The proof of the existence theorem for the minimal embedded resolution of a projective plane curve implies the following theorem:

THEOREM. Each connected component of $\mathcal{A}-\mathcal{A}_{\infty}$ is a bamboo which contains a unique dicritical component of $\varphi$ and this dicritical component is the only irreducible component of the bamboo which meets $\mathcal{A}_{\infty}$.

According to Orevkov (see [O] Lemma 2.1), this last theorem was already observed by Vitushkin.

Remark. Let $\mathcal{B}$ be a bamboo of $\mathcal{A}-\mathcal{A}_{\infty}$ and let $D_{\mathcal{B}}$ be its dicritical component. If $\mathcal{B}$ has more than one component, the components of $\mathcal{B}$ other than $D_{\mathcal{B}}$ define a sub-bamboo $\mathcal{B}^{\prime}$ of $\mathcal{B}$. The restricition to $\mathcal{B}^{\prime}$ of the function $\varphi$ is a finite constant. This value will be called the atypical value of the bamboo $\mathcal{B}$.

\section{Results.}

Consider a polynomial function $f$ of degree $d$ defined on $\mathbf{C}^{2}$. We assume that $f$ has no critical point. We shall call Jacobian pair a pair $(f, g)$ of polynomial functions defined on $\mathbf{C}^{2}$ for which the Jacobian is a non-zero constant. We shall say that $f$ is a Jacobian polynomial, if there is a polynomial $g$ such that $(f, g)$ is a Jacobian pair.

The conjecture stated above can be reformulated as follows. It is equivalent to the Jacobian conjecture:

ConjeCtURE. If $f$ is not a locally trivial topological fibration (on $\mathbf{C}$ ), it cannot be a Jacobıan polynomial. 
The Theorem of Hà and Lê can be translated in the following way: $\begin{aligned} I(f)= & \{\text { critical values } \neq \infty \text { of the restriction of } \varphi \text { to dicritical components }\} \cup \\ & \left.\text { atypical values of } \varphi \text { on each bamboos of } \mathcal{A}-\mathcal{A}_{\infty} \text { with at least two vertices }\right\}\end{aligned}$ This new formulation of the Theorem of Hà and Lê implies the following theorem:

THEOREM. Let $f$ be a complex polynomial function on $\mathbf{C}^{2}$ and let $\varphi$ be the minımal compactification of $f$. Assume that $f$ has no critical point. Then, $f$ is not a locally trivial fibration on $\mathbf{C}$ if and only if, either there is a bamboo of $\mathcal{A}-\mathcal{A}_{\infty}$ with at least two components or all bamboos in $\mathcal{A}-\mathcal{A}_{\infty}$ only contain one component and the restriction of $\varphi$ to at least one of them has critical points.

Therefore in an attempt to prove our conjecture above, we shall consider two cases:

CASE 1. In the graph $\mathcal{A}-\mathcal{A}_{\infty}$ there is at least one connected component with two vertices;

CASE 2. In the graph $\mathcal{A}-\mathcal{A}_{\infty}$ all the connected components have only one vertex (which is a dicritical component of $\varphi$ ) and the restriction of $\varphi$ on at least one dicritical component has degree strictly greater than one.

Examples. The function $X-X^{2} Y$ corresponds to the case 1 . The function $X-X^{4} Y^{4}$ belongs to the case 2 .

Now let $g$ be another complex polynomial function of degree $d^{\prime}$. Denote by $G$ the homogenized polynomial associated to $g$. Then, in order to prove our conjecture, the cases 1 and 2 subdivide in subcases, depending on the behaviour of the rational function $G / T^{d^{\prime}}$.

CASE 1. Let us call $D_{0}, D_{1}, \ldots, D_{r}$ the ordered components in a bamboo of $\mathcal{A}-\mathcal{A}_{\infty}$ with at least two vertices $(r \geqq 1), D_{0}$ being the dicritical component.

The case 1 subdivides in the following subcases:

a) The rational function $G / T^{d^{\prime}}$ is defined in some neighbourhood $\mathcal{U}$ of $D_{1} \cup \ldots \cup D_{r}$ :

i) The rational function $G / T^{d^{\prime}}$ is constant on $D_{1}, \ldots, D_{r}$ and $D \cap \mathcal{U}$;

ii) There is a $D_{i}$, with $1 \leqq i \leqq r-1$, which is dicritical for $G / T^{d^{\prime}}$;

iii) The component $D_{r}$ is dicritical for $G / T^{d^{\prime}}$;

iv) The component $D_{0}$ is dicritical for $G / T^{d^{\prime}}$;

b) There are points $x_{1}, \ldots, x_{s}$ in $D_{1} \cup \ldots \cup D_{r}$ where $G / T^{d^{\prime}}$ is not defined:

i) $s \geqq 2$;

ii) $s=1$ and $x_{1}$ is not on $D_{r}-\cup_{j=1}^{r-1} D_{j}$;

iii) $s=1$ and $x_{1}$ is on $D_{r}-\cup_{j=1}^{r-1} D_{j}$;

CASE 2. We first have the following result:

Proposition. Assume that $f$ has no critical point and that $f$ is not a locally trivial fibration. If all the connected components of $\mathcal{A}-\mathcal{A}_{\infty}$ contain only one vertex, there is at least one dicritical component $D$ of $\varphi$ on which $\varphi$ has a critıcal point. 
Proof. If $f$ is not a locally trivial fibration, the translation of the Theorem of Hà and Lê given above shows that under the assumptions of the proposition, the restriction of $\varphi$ to one of the dicritical components of $\varphi$ is critical at some point of this dicritical component. The proposition asserts that there is at least one dicritical component of $\varphi$ which carries a critical point of $\varphi$ itself and not its restriction.

Assume that $\varphi$ has no critical point. Then, Ehresmann Lemma implies that $\varphi$ is a locally trivial fibration on $\mathbf{C}$. This would imply that the Euler characteristic of the general fiber of $\varphi$ equals $1+\ell$, where $\ell$ is the number of dicritical components of $\varphi$. Therefore necessarily $\ell=1$. A result by T.T. Moh ([Mo]) interpreted in our setting implies that the restriction of $\varphi$ to this unique component must be of degree 1 , which would contradict the fact that this restriction has a critical point on this component.

Indeed, a direct proof of this theorem of T.T. Moh is possible using the connectedness theorem and a little topological argument.

Now consider a dicritical component $D_{0}$ of $\varphi$ on which $\varphi$ has a critical point $x$.

The case 2 subdivides in the following subcases:

a) The rational function $G / T^{d^{\prime}}$ is defined in some neighbourhood $\mathcal{U}$ of $D_{0}$ :

i) The rational function $G / T^{d^{\prime}}$ is constant on $D_{0}$;

ii) The component $D_{0}$ is dicritical for $G / T^{d^{\prime}}$;

b) There are points $x_{1}, \ldots, x_{s}$ in $D_{0}$ where $G / T^{d^{\prime}}$ is not defined:

i) none of these points are equal to $x$;

ii) one of them, say $x_{1}$ is actually $x$.

We can prove that, if $f$ has no critical point and is not a locally trivial fibration, for any polynomial function $g$ on $\mathbf{C}^{2}$, the pair $(f, g)$ is not a Jacobian pair in all the preceeding cases except the cases, case $1 \mathrm{a}$ iv) and case 2 a ii). In the unsolved case 1 a iv), we can conclude if we know that one of the restrictions of $\varphi$ or $\psi:=G / T^{d^{\prime}}$ on the dicritical component $D_{0}$ has degree one.

We now express our results differently. Let us say that a dicritical component of $\varphi$ is non-equisingular if $D_{0}$ belongs to a bamboo in $\mathcal{A}-\mathcal{A}_{\infty}$ of length at least two or if the restriction of $\varphi$ to $D_{0}$ has at least one critical point. Let us say that $D_{0}$ is strongly non-equisingular if $D_{0}$ belongs to a bamboo in $\mathcal{A}-\mathcal{A}_{\infty}$ of length at least two or if $\varphi$ has a critical point on $D_{0}$.

The above proposition says that if $C(f)=\emptyset$ and $I(f) \neq \emptyset$, then $\varphi$ has at least one strongly non-equisingular component.

The results we obtained so far in our attempt to prove the Jacobian Conjecture can be summarize as follows:

MaIn Theorem. Let $f$ be a polynomial such that $C(f)=\emptyset$ and $I(f) \neq \emptyset$. Let $g$ be a polynomial with $C(g)=\emptyset$. Then the pair $(f, g)$ cannot be a Jacobran pair if at least one of the following conditions is fulfilled:

1. There exists a strongly non-equisingular component of $\varphi$ which is not dicritical for $\psi$;

2. There exists a strongly non-equısıngular dicritıcal component $D_{0}$ for $\varphi$ for which the restriction $\varphi \mid D_{0}$ or $\psi \mid D_{0}$ has degree one.

The following proposition is not difficult but very useful 
Proposition. Let $(f, g)$ be a Jacobian pair. Let $\varpi: \mathcal{Z} \rightarrow \mathbf{P}^{2}$ be the composition of a finite sequence of point blowing-ups centered above the line at infinity $T=0$, such that $F / T^{d} \circ \varpi$ and $G / T^{d^{\prime}} \circ \varpi$ define morphisms $\varphi$ and $\psi$ from $\mathcal{Z}$ to $\mathbf{P}^{1}$. Then, the support of the divisor of the 2 -form $d \varphi \wedge d \psi$ is contained in $D_{\infty}(\mathcal{Z})=\varpi^{-1}(T=0)$.

In other words, the divisor of $d \varphi \wedge d \psi$ is a canonical divisor for $\mathcal{Z}$ which is confined at infinity (i.e. on $D_{\infty}(\mathcal{Z})$ ). The multiplicities of such a canonical divisor are well defined and can be computed from the sequence of blowing-ups. They do not depend on $\varphi$ and $\psi$.

For instance, this proposition implies that, in the case 1 above, if the dicritical component $D_{0}$ of $\varphi$ in $\mathcal{X}$ has a negative multiplicity in the canonical divisor of $\mathcal{X}$ concentrated at infinity, its strict transform $D_{0}^{\prime}$ in $\mathcal{Z}$ has the same negative multiplicity in the canonical divisor of $\mathcal{Z}$ concentrated at infinity. This implies that $\psi$ has a pole along $D_{0}^{\prime}$. Therefore $D_{0}$ cannot be a common dicritical component of $\varphi$ and $\psi$, which means that we are in one of the cases where our conjecture is true.

As an example, these observations applied to the case of $f(X, Y)=X-X^{2} Y$ show that it is not a Jacobian polynomial. The same argument works to show that many polynomials with no critical points cannot be Jacobian polynomials.

This leads to the following corollary of the Main Theorem:

COROLlary. Let $f: \mathbf{C}^{2} \rightarrow \mathbf{C}$ be a polynomial function without critical point. Then $f$ cannot be a Jacobian polynomial if there exists a dicritical component $D_{0}$ of $\varphi$ in $\mathcal{X}$ which is strongly non-equisingular and such that the multiplicity of $D_{0}$ in the canonical divisor of $\mathcal{X}$ confined at infinity either 1) is strictly negative or 2) is positıve and the restriction of $\varphi \mid D_{0}$ is of degree one.

\section{References}

[A] E. Artal, Une démonstration Géométrique du théorème d'Abhyankar-Moh, Preprint.

[A-M] S. Abhyankar, T.T. Moh, Embeddings of the line in the plane, Jr. Angw. Reine Math. 276 (1975), 148-166.

[B-C-W] H. Bass, E. H. Connell, D. Wright, The Jacobian Conjecture: reduction of degree and formal expansion of the inverse, Bull. Amer. Math. Soc. 7, (1982), 287-330.

[B] S.A. Broughton, Milnor numbers and the topology of polynomial hypersurfaces, Inv. Math. 92 (1988), 217-241.

[H-L] Hà Huy Vui, Lê Dũng Tráng, Sur la topologie des polynômes complexes, Acta Math. Vietn. 9 (1984), 21-32.

[L-M-W] Lê Dũng Tráng, F. Michel, C. Weber, Sur le comportement des polaires associées aux germes de courbes planes, Comp. Math. 72 (1989), 87-113.

[Mo] T.T. Moh, On analytic irreducibility at $\infty$ of a pencil of curves, Proc. Amer. Math. Soc. 44 (1974), 22-24.

[Mu] D. Mumford, Algebraic Geometry I, Complex Algebraic Varieties, Grundlehren der math. Wissenschaften 221, Springer Verlag (1976).

[O] S. Yu. Orevkov, On three-sheeted polynomial mappings of $\mathbf{C}^{2}$, Math. USSR Izvestiya, 29 (1987), 587-596.

[V] A. G. Vitushkin, Certain examples in connections with problems about polynomial transfor- 
mations of $\mathbf{C}^{n}$, Math. USSR Izvestiya, 5 (1971), 278-288.

LÊ DŨNG TRÁNG

UFR DE MathÉMATIQUES ET INFORMATIQUe

Université de Provence

F-13003 Marseille, France

C. Weber

INSTITUT DE MathÉMATIQUES

2-4 RuE DU LIÈvRE

Case Postale 240

CH-1211 GENÈVE 24, SUISSE 